REGARDS

SUR L'ECONOMIE ALLEMAND

BULLETIN ECONOMIQUE DU CIRAC
Regards sur l'économie allemande

Bulletin économique du CIRAC

$67 \mid 2004$

Varia

\title{
Une convalescence fragile
}

Isabelle Bourgeois

\section{(2) OpenEdition}

Journals

Édition électronique

URL : http://journals.openedition.org/rea/3792

DOI : $10.4000 /$ rea.3792

ISBN : 978-2-8218-0830-0

ISSN : 1965-0787

Éditeur

CIRAC

Édition imprimée

Date de publication : 1 juillet 2004

Pagination : 3-6

ISSN : 1156-8992

Référence électronique

Isabelle Bourgeois, « Une convalescence fragile », Regards sur l'économie allemande [En ligne], 67 | juillet 2004, mis en ligne le 08 octobre 2009, consulté le 15 septembre 2020. URL : http:// journals.openedition.org/rea/3792 


\section{Une convalescence fragile}

La reprise annoncée se confirme. Mais elle restera modeste, avec une croissance de $1,5 \%$ en 2004 comme en 2005, selon le Rapport de printemps des Instituts de conjoncture publié en avril. Depuis l'automne en effet, production et demande intérieure ne cessent d'augmenter, légèrement encore certes, mais continûment. La production est tirée par une conjoncture mondiale favorable malgré la forte appréciation de l'euro face au dollar, et qui bénéficie à l'ensemble de la zone euro au même titre que la politique monétaire expansive de la BCE qui entretient un environnement propice aux activités. Dopés par l'embellie extérieure et les perspectives qu'elle offre, les investissements en biens d'équipement ont redémarré dès la fin 2003, évoluant ainsi positivement pour la première fois depuis trois ans. L'économie allemande est bel et bien sortie de la phase de stagnation - grâce aux seules exportations une fois de plus, car la dynamique reste bridée par l'attentisme intérieur.

Depuis la publication du rapport, le boom des exportations sur les quatre premiers mois de l'année et, à leur suite, celui de la production industrielle, en particulier des biens intermédiaires, d'investissement, mais aussi de consommation, confirme cette lecture. Selon les estimations publiées par l'institut ifo (Munich) le 22 juin, le PIB devrait avoir augmenté de 1,6\% au premier semestre 2004 par rapport à la même période 2003. Ce résultat meilleur que prévu amène dès lors l'institut à revoir légèrement à la hausse ses prévisions pour 2004, à l'instar de ses homologues. Désormais, toutes oscillent entre $1,5 \%$ et $1,8 \%$. Ces taux incluent un effet calendrier non négligeable (0,5 point), 2004 ayant peu de jours fériés. Mais dès l'an prochain, l'embellie sera moins nette : le nombre de jours ouvrables baisse à nouveau et, surtout, les répercussions sur la zone euro du ralentissement conjoncturel attendu en Chine et aux USA devraient amoindrir la vigueur de l'export. Les prévisions pour 2005 varient dès lors entre 1,3\% (IfW) et 2,0\% (RWI), du fait des incertitudes liées à l'évolution des prix des matières premières et du pétrole brut et aux décisions politiques à venir sur la rigueur budgétaire et la poursuite des réformes structurelles.

En 2003, les exportations à destination de l'UE (56,4\% du total de l'export allemand) ont été particulièrement dynamiques: avec un volume de 357 milliards $€$, elles contribuaient au PIB à hauteur de 17,4\% (en valeur nominale). Par destinations, la part de l'UE progressait d'un point de pourcentage par rapport à 2002, celle de l'Amérique du Nord perdant l'équivalent pour tomber à 11,0\%. La part des PECO a continué de progresser (10,7 \% après 10,2 \%) à haut niveau, l'Allemagne ayant considérablement intensifié ses échanges surtout avec les nouveaux Etats membres au cours des dix dernières années. Celle de l'Asie du Sud-Est, dont la Chine, passait de $8,0 \%$ à $8,4 \%$. Cette structure révèle les forces et faiblesses du moteur conjoncturel allemand. D'abord, la dépendance relative d'une conjoncture américaine qui donne des signes d'essoufflement. Ensuite, le positionnement solide sur des marchés en transition, et donc en forte croissance comme l'Europe de l'Est et la Chine, mais avec les risques inhérents de surchauffe (Chine). Enfin, l'étroite dépendance du marché européen, principalement de la zone euro, prémunit certes l'économie allemande comme celle des autres Etats membres contre les risques émanant des variations du taux de change $€ / \$$. Mais que l'économie de la zone euro donne des signes de faiblesse comme c'est le cas actuellement (croissance de $+0,4 \%$ seulement en 2003 , de $1,6 \%$ en 2004 et de $2,0 \%$ en 2005 selon les Instituts), et la conjoncture allemande s'en ressent.

La forte appréciation de l'euro face au dollar au début de l'année n'aura finalement guère pénalisé les exportations allemandes qui ont par ailleurs bénéficié d'une inflation modérée. Les exportateurs, qui avaient compensé l'effet change en comprimant leurs marges, devraient retrouver une plus grande latitude dans la fixation des prix aujourd'hui que l'envolée de l'euro a pris fin, rappellent les Instituts. Or s'ils ont pu adapter leurs prix à l'export, c'est que la compétitivité prix allemande est supérieure à celle des autres membres de la zone euro, explique la Bundesbank (rapport de juin 2004). Le taux d'inflation étant particulièrement faible en Allemagne, cette compétitivité a augmenté continûment depuis l'introduction de la monnaie européenne. C'est là une explication de l'excellente tenue de l'export allemand, et elle
Une reprise confirmée, mais modeste...

... à la traîne de l'économie mondiale et européenne

Plus de $56 \%$

de l'export vers l'UE

Une balance commerciale toujours excédentaire 
Les investissements redémarrent

La production de biens intermédiaires se renforce

Des facteurs exceptionnels ont ralenti la décrue du BTP en 2003

Emploi : pas d'amélioration avant 2005 incite à l'optimisme quant à son évolution future. L'envolée de l'euro face au dollar a eu pour autre effet de faire baisser les prix à l'import, ce qui a compensé la flambée des prix des matières premières et soutenu le frémissement de la demande intérieure. Au total, la balance commerciale sera largement positive une fois de plus cette année. Elle pourrait être légèrement négative l'an prochain, selon l'institut ifo, la consommation privée devant redémarrer $(+1,5 \%)$, tirant les importations $(+6 \%$ environ) alors que la croissance des exportations ralentit un peu $(+5,1 \%$ au lieu de $7,9 \%$ cette année).

La bonne tenue du marché mondial et les perspectives de rendement qu'elle offre amènent les entreprises à accroître à nouveau leurs capacités en réaction à des carnets de commandes bien remplis. Les investissements en biens d'équipement ont donc repris, rompant avec un recul de trois ans. Mais la dynamique reste bridée par la timidité des commandes domestiques de biens d'investissement dans la mesure où le taux d'utilisation des capacités demeure faible et que le cadre réglementaire des activités s'est détérioré (modification du régime des amortissements, réduction des subventions et dégrèvements fiscaux). Mais au fil des mois, la reprise des investissements devrait s'affermir avec l'amélioration des perspectives sur un marché domestique où la demande semble vouloir redémarrer. S'y ajoutent une relative stabilité des coûts salariaux, ainsi qu'un contexte plus favorable au financement des activités (taux d'intérêt avantageux sur le long terme, retour de l'optimisme sur les marchés financiers). Corrigée de l'effet calendrier, la hausse des investissements bruts d'équipement et autres biens sera de 1,4\% cette année (hors correction : 3,2 \%) et de 5,4\% en 2005 (Instituts).

Hors BTP, la production industrielle redémarre. Elle devrait croître de 1,6 \% sur l'ensemble de l'année 2004. C'est peu, mais elle a presque stagné au premier trimestre $(+0,3 \%)$ par rapport à un dernier trimestre 2003 où elle avait augmenté de 2,1\%. Ces fortes variations expriment les doutes des industriels quant à la vigueur de la reprise, nourris par une molle demande intérieure et l'incertitude sur l'évolution des prix des matières premières (DIW Wochenbericht 25/04). Néanmoins, depuis le début de l'année, la tendance est à la hausse des activités, dans les branches productrices de biens d'investissement, mais aussi de biens de consommation. La demande provient pour l'essentiel de l'étranger, dopant la production de biens intermédiaires, en hausse de 2,6\% au premier trimestre 2004. L'un des principaux piliers de l'industrie allemande (avec un taux d'exportation de $39 \%$ ) semble donc avoir surmonté l'accès de faiblesse de ces deux dernières années.

Le BTP poursuit sa décrue, exceptionnellement ralentie en 2003. Dans l'habitat, les ménages avaient anticipé leurs investissements en prévision de l'abrogation des aides à l'accession à la propriété projetée par le gouvernement fédéral pour contrefinancer la baisse de l'impôt sur le revenu. Mais la hausse est relative, les ménages hésitant à s'engager alors que leurs revenus réels stagnent et que les tensions sur le marché de l'emploi persistent. Dans le BTP industriel, quelques grands projets, ainsi que les travaux de reconstruction (infrastructures) engagés à la suite des inondations de l'été 2002 dans l'est du pays, masquent l'ampleur réelle du recul de l'activité. Aucune amélioration n'est en vue à moyen terme, les collectivités, déficitaires, n'investissant que très modérément. Au total, le BTP sera en baisse de $-0,8 \%$ cette année, hors effet calendrier (sans correction : +1,0\%, Instituts).

Après trois ans de stagnation, le marché de l'emploi reste tendu. Le nombre d'actifs occupés a continué de baisser en 2003 , sous l'effet de l'engouement pour les Ich$A G$ et, plus encore, pour les mini-jobs et autres 'petits boulots' (voir dans ce numéro). Si la progression du chômage s'est ralentie, cela est dû pour l'essentiel à une révision des statistiques (-150 000 chômeurs inscrits selon les Instituts), à l'accélération de la rotation chômage-emploi et à des conditions plus restrictives depuis l'entrée en vigueur des premières mesures Hartz. Sans ces mesures dont les effets se poursuivent cette année, le nombre de chômeurs s'établirait à 4,6 millions en moyenne sur 2004, rappelle l'institut ifo. Car l'emploi soumis à cotisations sociales n'évolue pas, les entreprises hésitant à embaucher dans un contexte de reprise encore fragile et parce que les coûts salariaux leur semblant de plus en plus élevés en comparaison des tarifs en vigueur dans les autres Etats membres, surtout les dix nouveaux. Cette évolution n'est pas propre à l'Allemagne, dont le taux de chômage 
harmonisé selon les données de l'OIT (9,3\% en 2003) n'est supérieur que d'un demi point à la moyenne de la zone euro (8,8\% ; France : 9,4\%). Une légère décrue n'est attendue qu'avec le retour d'une croissance plus vigoureuse.

La consommation des ménages n'a pas décollé au dernier trimestre 2003, contrairement à l'effet espéré de la récente baisse de l'impôt sur le revenu (voir REA $65 / 04)$. Le coup de pouce au revenu disponible a été absorbé par la hausse des coûts de santé et par un taux d'inflation qui dépasse $2 \%$ depuis le mois de mai, suite à la flambée des prix pétroliers et à l'entrée en vigueur de la dernière tranche de l'écotaxe. En l'absence de certitudes sur l'évolution économique et l'avenir de leurs retraites, les ménages maintiennent d'autant plus volontiers leur épargne de précaution (voir dans ce numéro) que, au mieux, leur revenu disponible réel stagne (+0,4\% en 2004 , Instituts). Ils semblent avoir compris confusément une tendance que l'IfW de Kiel décrit ainsi : « dans dix ans, la majorité de la population ... aura un standard de vie inférieur à ce qu'il est aujourd'hui » (Handelsblatt, 18-06-04).

Cette tendance qui résulte du vieillissement démographique est entretenue par une dynamique de croissance de plus en plus bridée depuis le début des années 90. Le taux d'investissement a fléchi, le volume des heures travaillées n'a cessé de diminuer, et la productivité du travail s'est ralentie, remarquent les Instituts. Les choix politiques sont en grand partie responsables de ces évolutions : les collectivités ont réduit leurs investissements, notamment au détriment du capital humain, amoindrissant d'autant le potentiel de productivité. La politique de l'emploi et de fixation des salaires, le subventionnement des préretraites, ont eu des effets désincitatifs sur l'activité et dévalorisé le travail. Dans ces conditions, définir et mener une politique économique en faveur d'une croissance durable n'est guère facile, l'économie allemande se trouvant prise dans un engrenage où l'explosion des coûts sociaux (chômage, transferts liés à l'unification) creuse la dette publique, limitant la réduction des prélèvements obligatoires, pourtant indispensable pour maintenir l'activité et l'emploi dans les frontières nationales, et entravant l'investissement dans la compétitivité du capital humain, qui devrait être la priorité d'un site vieillissant affrontant la concurrence directe de jeunes économies dynamiques.

Pour sortir de cet engrenage, le gouvernement Schröder avait amorcé des réformes structurelles (Agenda 2010). Ce début courageux avait rendu confiance aux acteurs économiques, c'était le plus urgent pour briser leur attentisme. Un an après, l'impact des réformes est décevant. Mais elles ne produiront leurs effets que sur le long terme et que si elles sont poursuivies plus avant, rappellent une fois de plus les Instituts. Or les citoyens commencent à grogner. Et la crise politique au sein de la coalition gouvernementale comme le tir nourri de l'opposition, avivés par la campagne électorale permanente de l'année 2004, les désorientent encore un peu plus. La consommation des ménages s'en ressent. Le camouflet au parti SPD lors des européennes a fragilisé la position du chancelier. Le seul soutien qu'il puisse attendre en ce début d'été provient des acteurs et observateurs économiques qui veillent à entretenir l'ardeur réformiste du gouvernement et, partant, le moral des milieux d'affaires. Dans ce contexte, l'élection d'un nouveau président de la République issu de l'économie, en la personne de Horst Köhler, ex-président du FMI, est un gage supplémentaire pour une politique de réformes consistante.

L'efficacité des réformes passe par la consolidation budgétaire : la dégradation persistante des finances publiques allemandes n'inquiète pas seulement la Commission européenne, elle alarme tous les économistes puisqu'elle obère la croissance future. Avec une dette qui s'élèvera cette année à $66 \%$ du PIB, l'Allemagne est surendettée, et les pouvoirs publics ont épuisé leur marge de manœuvre. Depuis 3 ans, l'Allemagne ne respecte plus les critères de Maastricht. Or elle s'était engagée à le faire en 2005 en réduisant le déficit structurel de 0,6 point de PIB cette année et d'au moins 0,5 point l'an prochain. Ce dernier varie, selon les sources, entre 2,3\% et $3,5 \%$ du PIB (2003). La faiblesse conjoncturelle est donc moins en cause que les dérives sociales, les subventions indifférenciées, le coût du fédéralisme ou cette gestion administrative peu rigoureuse qu'incrimine la Cour fédérale des Comptes.

La rigueur s'impose. Le ministre fédéral des Finances a présenté le 23 juin un budget fédéral 2005 « respectueux de la conjoncture et de ferme consolidation » selon ses propres termes. Il fixe les dépenses du Bund à 258,3 milliards $€$, soit une
Déprimés et désorientés, les ménages consomment peu

Une politique économique difficile à mener

Poursuivre les réformes structurelles

Et consolider les finances publiques

Un budget « respectueux de la conjoncture et de ferme consolidation " 
hausse de 1,1\% qui s'explique par l'explosion des budgets sociaux. Mais les recettes fiscales sont en baisse, et le Bund doit recourir à la dette. Pour ne pas dépasser les dépenses d'investissement $(22,8$ milliards $€)$ et respecter le critère de stabilité de la Loi fondamentale, l'endettement est fixé à 22 milliards $€$. Soit 15,5 milliards $€$ de moins que les besoins réels. Le Bund envisage de combler cet écart grâce à la vente de participations (dont le restant des actions de Deutsche Post et Deutsche Telekom) et à la réduction de subventions - dont ces primes d'accession à la propriété que les Länder se refusent d'abroger au Bundesrat. Si Hans Eichel a demandé à tous les ministères de comprimer leurs budgets, il se refuse pour l'instant à réduire encore plus les dépenses. Les économistes critiquent vertement ce budget très hypothétique, estimant, à l'instar du président de l'institut ifo, que l'Allemagne devient ainsi « la risée de l'Europe » (Handelsblatt, 23-06-04).

COMMENT FAIRE POUR ASSAINIR LES FINANCES PUBLIQUES sans étouffer la timide reprise ? Comment respecter le court terme (les critères de Maastricht) et le long terme (le retour d'une croissance durable) ? Ce dilemme a déclenché une polémique où s'affrontent avocats d'une politique de relance financée à crédit (parmi eux, la base du SPD et les syndicats) et tenants d'un strict respect des critères de stabilité nationaux et européens (dont tous les Instituts, sauf le neo-keynesien DIW). Pour ne pas mettre en danger la poursuite des réformes structurelles, le gouvernement fédéral a cherché un compromis visant à ménager difficultés conjoncturelles et impératifs de rigueur - en attendant aussi que se clarifie à l'échelon européen l'interprétation à donner au Pacte de Maastricht.

Isabelle Bourgeois (24-06-2004)

Prévisions du rapport de printemps 2004 des instituts de conjoncture

\begin{tabular}{|c|c|c|c|c|c|c|}
\hline & 2002 & 2003 & 2004 & Sem. 1 & Sem. 2 & 2005 \\
\hline \multicolumn{7}{|c|}{ (variation en \% par rapport à la même période de l'année précédente) } \\
\hline $\begin{array}{l}\text { PIB } \\
\text { Est } \\
\text { Ouest (Berlin inclus) }\end{array}$ & $\begin{array}{l}0,2 \\
0,1 \\
0,2\end{array}$ & $\begin{array}{r}-0,1 \\
0,2 \\
-0,1\end{array}$ & $\begin{array}{l}1,5 \\
1,3 \\
1,5\end{array}$ & 1,3 & 1,8 & $\begin{array}{l}1,5 \\
1,5 \\
1,5\end{array}$ \\
\hline $\begin{array}{l}\text { Consommation privée }{ }^{\text {a) }} \\
\text { Consommation publique }\end{array}$ & $\begin{array}{r}-1,0 \\
1,7\end{array}$ & $\begin{array}{r}-0,1 \\
0,9\end{array}$ & $\begin{array}{r}0,4 \\
-0,4\end{array}$ & $\begin{array}{l}-0,2 \\
-0,4\end{array}$ & $\begin{array}{r}0,9 \\
-0,4\end{array}$ & $\begin{array}{l}1,2 \\
0,3\end{array}$ \\
\hline $\begin{array}{l}\text { Investisssements bruts en biens d'équipt. } \\
\text { Equipement et autres biens } \\
\text { Construction }\end{array}$ & $\begin{array}{l}-6,7 \\
-7,6 \\
-5,8\end{array}$ & $\begin{array}{l}-2,9 \\
-2,3 \\
-3,4\end{array}$ & $\begin{array}{l}2,0 \\
3,2 \\
1,0\end{array}$ & $\begin{array}{l}1,8 \\
1,4 \\
2,1\end{array}$ & $\begin{array}{l}2,2 \\
4,8 \\
0,1\end{array}$ & $\begin{array}{r}2,3 \\
5,1 \\
-0,2\end{array}$ \\
\hline $\begin{array}{l}\text { Demande intérieure } \\
\text { Exportations } \\
\text { Importations }\end{array}$ & $\begin{array}{r}-1,6 \\
3,4 \\
-1,7\end{array}$ & $\begin{array}{l}0,3 \\
1,2 \\
2,6\end{array}$ & $\begin{array}{l}1,0 \\
6,6 \\
5,7\end{array}$ & $\begin{array}{l}0,3 \\
6,9 \\
4,6\end{array}$ & $\begin{array}{l}1,7 \\
6,3 \\
6,7\end{array}$ & $\begin{array}{l}1,3 \\
5,0 \\
4,9\end{array}$ \\
\hline $\begin{array}{l}\text { Revenu national brut } \\
\text { Rev. brut issu du travail salarié } \\
\text { Rev. brut issu des entr. et de la propté. }\end{array}$ & $\begin{array}{l}0,5 \\
0,8 \\
4,8\end{array}$ & $\begin{array}{r}0,4 \\
0,2 \\
-0,3\end{array}$ & $\begin{array}{l}2,3 \\
0,7 \\
6,4\end{array}$ & $\begin{array}{l}2,4 \\
0,5 \\
7,6\end{array}$ & $\begin{array}{l}2,2 \\
0,8 \\
5,1\end{array}$ & $\begin{array}{l}2,5 \\
1,3 \\
5,7\end{array}$ \\
\hline Revenu disponible & 0,5 & 0,9 & 1,6 & 1,0 & 2,3 & 2,4 \\
\hline Consommation des ménages & 0,3 & 0,9 & 1,6 & 0,9 & 2,2 & 2,5 \\
\hline Epargne des ménages & 3,8 & 3,4 & 2,3 & 1,7 & 3,1 & 2,2 \\
\hline Prix à la consommation $\left.{ }^{b}\right)$ & 1,4 & 1,1 & 1,3 & - & - & 1,2 \\
\hline Coûts salariaux unitaires & 0,7 & 0,6 & $-0,5$ & - & - & $-0,1$ \\
\hline \multicolumn{7}{|c|}{ (état) } \\
\hline $\begin{array}{l}\text { Déficit public en } \% \text { du PIB } \\
\text { Dette publique (en milliards } € \text { ) }\end{array}$ & $\begin{array}{r}-3,5 \\
-74,3\end{array}$ & $\begin{array}{r}-3,9 \\
-82,1\end{array}$ & $\begin{array}{l}-3,7 \\
-80,2\end{array}$ & - & $\begin{array}{l}- \\
-\end{array}$ & $\begin{array}{r}-3,5 \\
-78,6\end{array}$ \\
\hline $\begin{array}{l}\text { Actifs (en millions) } \\
\text { Est, Berlin inclus (en millions) }{ }^{\text {c) }} \\
\text { Ouest (en millions) }\end{array}$ & $\begin{array}{r}38,61 \\
7,61 \\
31,00\end{array}$ & $\begin{array}{r}38,19 \\
7,51 \\
30,68\end{array}$ & $\begin{array}{c}38,11 \\
7,47 \\
30,64\end{array}$ & $\begin{array}{l}- \\
-\end{array}$ & - & $\begin{array}{r}38,22 \\
7,43 \\
30,78\end{array}$ \\
\hline $\begin{array}{l}\text { Chômeurs (en millions) } \\
\text { Est, Berlin inclus (en millions) } \\
\text { Ouest (en millions) }\end{array}$ & $\begin{array}{l}4,06 \\
1,56 \\
2,50\end{array}$ & $\begin{array}{l}4,38 \\
1,62 \\
2,75\end{array}$ & $\begin{array}{l}4,33 \\
1,58 \\
2,75\end{array}$ & $\begin{array}{l}- \\
-\end{array}$ & $\begin{array}{l}- \\
-\end{array}$ & $\begin{array}{l}4,28 \\
1,57 \\
2,70\end{array}$ \\
\hline $\begin{array}{l}\text { Taux de chômage (en \%) } \\
\text { Est, Berlin inclus } \\
\text { Ouest }\end{array}$ & $\begin{array}{r}9,5 \\
17,0 \\
7,5\end{array}$ & $\begin{array}{r}10,3 \\
17,8 \\
8,2\end{array}$ & $\begin{array}{c}10,2 \\
17,5 \\
8,2\end{array}$ & $\begin{array}{l}- \\
-\end{array}$ & $\begin{array}{l}- \\
-\end{array}$ & $\begin{array}{r}10,1 \\
17,5 \\
8,1\end{array}$ \\
\hline Taux d'épargne & 10,6 & 10,8 & 10,9 & 12,4 & 9,4 & 10,9 \\
\hline
\end{tabular}

Source des données : Rapport de printemps des six instituts de conjoncture DIW (Berlin), HWWA (Hambourg), ifo (Munich), IfW (Kiel), IWH (Halle), RWI (Essen), en date du 23-04-2004. Données établies selon le Système européen des comptes nationaux (SEC 95) ; 2004 et 2005 ; prévisions des Instituts. a) ménages et organismes privés à but non lucratif ; b) aux prix de 1995 ; c) les données relatives au marché de l'emploi émanent de l'Office fédéral des Statistiques. Sur la différence avec les données OIT (1 point de pourcentage environ), voir REA 59/02. 